\title{
The neuroactivation of cognitive processes investigated with SPECT
}

\author{
Daniela Montaldi* and Andrew R. Mayes \\ Department of Psychology, University of Liverpool, \\ $U K$
}

The last ten years have seen the development and expansion of an exciting new field of neuroscientific research; functional mapping of the human brain. Whilst many of the questions addressed by this area of research could be answered using SPECT, relatively few SPECT activation studies of this kind have been carried out. The present paper combines an evaluation of SPECT procedures used for neuroactivation studies, and their comparison with other imaging modalities (i.e., PET and fMRI), with a review of SPECT neuroactivation studies that yield information concerning normal brain function with a particular emphasis on the brain activations produced by memory processing. The paper aims to describe and counter common misunderstandings regarding potential limitations of the SPECT technique, to explain and illustrate which SPECT procedures best fulfill the requirements of a neuroactivation study, and how best to obtain information about normal brain function (whether using normal healthy subjects or patients) and finally to highlight SPECT's potential future role in the functional mapping of the human brain.

Keywords: SPECT, neuroactivation, memory, cerebral blood flow, ${ }^{99 \mathrm{~m}}$ Tc HMPAO

\section{Introduction}

Over the past decade cognitive neuroactivation research has become one of the most rapidly expanding fields of neuroscience. This rapid expansion has occurred because technological developments have made it possible to image indirectly the neural correlates of cognition. Until recently the majority of neuroactivation studies were carried out using Positron Emission Tomography (PET). However, developments with

* Corresponding author: Daniela Montaldi, Department of Psychology, University of Liverpool, PO box 147, Liverpool, L69 3BX, UK. Tel.: +44 151794 5629; Fax: +44 151794 5635; E-mail: d.montaldi@liverpool.ac.uk. functional applications of magnetic resonance imaging (fMRI) have led to fMRI becoming the dominant functional neuroimaging modality. Despite the excitement growing within neuroactivation research, surprisingly few SPECT neuroactivation studies have been published. Two factors probably underlie the scarcity of published SPECT neuroactivation studies. First, there is a widespread belief that PET has several advantages over SPECT as a neuroactivation technique. Second, and probably related to this belief, there is a cultural difference such that SPECT has primarily been funded as a tool for clinical investigation whereas PET is more often funded as a research tool, particularly for neuroactivation research.

We shall argue in this paper that closer inspection of the evidence indicates that these advantages are less striking, general, or genuine than many workers believe. Furthermore, there are some genuine advantages of SPECT over PET, which are appreciated by relatively few. Together these widespread misconceptions have probably unjustifiably discouraged many from applying this imaging modality to cognitive neuroactivation research. The relative advantages and disadvantages of PET, SPECT, and fMRI will be discussed below. We will first consider what are widely believed to be the advantages of PET relative to SPECT as a neuroactivation technique. Then, we will consider what may be the advantages of SPECT over PET as a neuroactivation technique. Finally, we will briefly contrast fMRI and emission tomography and consider the advantages and disadvantages of fMRI relative to the two emission tomography techniques. Following this, we discuss SPECT neuroactivation procedures and how these have been used before concluding with a discussion and evaluation of SPECT's potential contribution to this area of neuroscience. 


\section{Comparison of PET and SPECT as neuroactivation techniques}

\subsection{Possible advantages of PET over SPECT}

The first of the advantages that Emission Tomography studies using PET have long been considered to have over those using SPECT is that the short half-life of the radiopharmaceuticals used with PET (typically a few minutes) allows several different cognitive conditions to be imaged in one session. A complete experiment can, therefore, be easily carried out without subjects needing to be repositioned. In contrast, as the half-life of the radiopharmaceuticals used with SPECT is generally much longer, different cognitive conditions are best imaged on different days. This design requires that subjects need to attend for more than one scanning session and the sessions must be at least 24 hours apart. It is widely believed that since the subjects must be repositioned each time, the successful use of the procedure is more dependent on the co-registration of scans, and hence may be liable to error resulting from incorrect co-registration. PET as well as SPECT neuroactivation studies require that images acquired at different times are co-registered. As PET scans are usually acquired over a period of around an hour, there is likely to be considerably less positional change than will be the case with SPECT scans that are acquired days apart so that subjects will have moved out of and back into the scanner between scans. However, the belief that this difference between SPECT and PET causes a greater problem for SPECT is based on a misconception because, as long as steps are taken to minimise excessive repositioning variation, co-registration accuracy should not depend on the extent of positional change. What is difficult is the very fine co-registration of images and error here arises for reasons unconnected with the initial amount of separation of images. In other words, the problem can be the same for PET and SPECT.

A second advantage that PET is widely believed to have relative to SPECT is that the nature of the radiopharmaceuticals used with it allows a greater number of scans to be performed with each PET experiment. This means that, relative to SPECT, PET studies can typically compare a greater number of conditions within a single session and can also replicate conditions within a single session. However, although it is clearly advantageous to be able to compare more conditions within an experiment, it is more doubtful that being able to replicate the same condition within a single PET session confers any real advantage over SPECT. As will be discussed below, the quality of the data that can be extracted from a single SPECT scan matches that which can be extracted from two or even three PET scans. This means that each condition probably has to be run three times for PET activation studies, but only once for SPECT activation studies in order to obtain similar quality images. So a typical PET study that has 12 conditions should actually include two repetitions of each condition, which means that only four different cognitive conditions will be included. Although SPECT activation studies have until recently only included two different conditions, it has now been shown that four condition studies can be undertaken [1]. There is also a potential psychological problem with repeating conditions because repetition of what is nominally the same task may well change the nature of the psychological processes engaged [25]. A better approach for PET studies might be to avoid repeating conditions unless there is a specific interest in the psychological changes that may be induced (for example, see [14]), and instead compensate for the relatively poor quality of the image data extractable from a single PET scan by including more subjects in each study.

Nearly everyone believes that PET neuroactivation studies have a third and very clear advantage over SPECT studies: better spatial resolution. This is certainly true of the optimal resolution that can be obtained from most PET scanners relative to standard clinical SPECT scanners, but there are nevertheless high quality dedicated brain-scanning SPECT systems, the spatial resolution of which is comparable to that of less sensitive PET systems. A perhaps more important qualification to the widely held belief that PET has greater spatial resolution than SPECT relates to the analysis procedures that are currently run with neuroactivation data, e.g., statistical parametric mapping (SPM) [7]. These procedures involve a smoothing stage that dramatically reduces the spatial resolution of the image in order to facilitate co-registration to Talaraich space [29]. It is probably the case that the spatial resolution of the analysed image is influenced by the analysis procedure much more than it is by the spatial resolution of the emission tomography system used to acquire the data (the same point applies to fMRI data).

\subsection{Possible Advantages of SPECT over PET}

It is less widely appreciated that SPECT techniques which use long half-life radiopharmaceuticals also have some advantages relative to PET. The first advantage is that the technology of SPECT allows the administra- 
tion of the radiopharmaceutical to be carried out away from the scanner in a different and more comfortable environment in which subjects are able to perform the selected cognitive tasks under more natural conditions. Following this, the scan can be acquired up to two hours later while the subjects rest or listen to music. This not only allows for testing to be carried out in an environment in which distraction can be kept at a very low level, but crucially removes the problem of limiting subject movement during task performance.

A second advantage of SPECT relative to PET is the ease with which patients can be examined in neuroactivation paradigms. This second advantage arises out of the first one because it is easier to test patients' performance of cognitive and motor tasks in the less constrained and less distracting environment that can be used in SPECT neuroactivation studies. It should be noted that for exactly the same reason the ability to use an unconstrained environment also gives SPECT an advantage over fMRI in patient work.

The third advantage of SPECT is that the temporal window of activation to which the technique is sensitive is around 40 seconds whereas that of PET is generally longer. This increases the ability to detect the activating effects of cognitive processes that operate optimally over short periods of time.

The fourth advantage of SPECT is that the half-life of the radiopharmaceuticals that can be used with SPECT allows for maximum acquisition of data for each scan. This has the important consequence that the quality of the data obtainable from one single SPECT scan at least matches that which can be obtained from two or even three PET scans because these have to be acquired much more rapidly. It is therefore possible, as discussed above, merely to run a cognitive condition once in order to get images of equivalent quality to those that can be obtained only with one or two repetitions if PET were to be used.

\section{Advantages and disadvantages of fMRI relative to emission tomography}

Apart from being a non-invasive imaging technique, fMRI has two other major advantages over emission tomography. First, it offers the possibility of much greater spatial resolution. This possibility has, however, still not been properly realized, partly because, as already indicated, current analysis procedures such as SPM reduce spatial resolution well below the levels that are determined by the physical constraints inherent in the imaging method. Even so, the physical constraints are less with fMRI and there is clearly greater potential for achieving higher spatial resolution with this technology. One of the ways in which this potential for achieving greater spatial resolution in the localization of activations is realised is through the accurate coregistration of functional scans to individual structural scans. Such accurate co-registration should be easier to achieve within one imaging modality rather than across imaging modalities as is essential if emission tomography techniques are used.

Second, fMRI acquires whole brain data very rapidly, thereby providing much greater temporal resolution as well as spatial resolution. Realisation of this potentially greater temporal resolution in imaging brain activations will be facilitated by the recent development of event-related recording procedures in which the temporal properties of the blood oxygenation level dependent (BOLD) response can be related to individual events or processes with precise onset times. Although the BOLD response takes several seconds to reach its peak value, it is possible to identify latency differences of the response under different conditions within one brain region very accurately. Comparisons across brain regions are problematic because it is currently not possible to determine whether differences are underlain primarily by variations in the timing of neural activity or whether vascular differences between the regions play a significant role. Whatever its value for determining the precise temporal properties of neural responses eventually turns out to be, event-related fMRI is alone among cognitive neuroactivation procedures in allowing researchers to determine the pattern of brain activation produced when individual and specific cognitive operations are performed. The eventrelated procedure also allows experimenters to present a random sequence of events of interest and so examine the brain activations produced by specific cognitive processes without the contaminating effects of strategic or mental set factors. This is not possible for emission tomography procedures.

While for these reasons, fMRI has fast become the principal imaging technique applied to neuroactivation research, it is not without its disadvantages. Even more than PET, the fMRI environment is distracting (with high noise levels), physically limiting (so that subjects have to remain very still whilst performing difficult mental tasks), and can be anxiety provoking. As a result, it is difficult to provide instructions and to monitor performance on many tasks, especially those which would normally require a spoken verbal output. It is, 
therefore, less suitable for patient work and in some cases also for work with healthy volunteers.

Although rapid developments in technology and image analysis will soon overcome problems relating to fMRI data acquisition, at present this is particularly vulnerable to artefact (motion and susceptibility artefact). At best, these artefacts require the application of correction techniques and at worst, in some brain regions (medial and inferior temporal, and orbitofrontal cortices), there might be failure to detect any BOLD signal whatsoever. Indeed, there is currently some debate as to whether susceptibility artefact might effectively "shift" an activation spatially, thus reducing fMRI's accuracy at spatially localising activations. It is clear, however, that despite current problems, fMRI will dominate this field of neuroscience, although there will remain roles for both PET and SPECT and an accurate understanding of the advantages, disadvantages and potential of each emission tomography technique will clarify their respective and overlapping roles.

In the rest of this paper, we first outline some of the different methods that have been used in SPECT neuroactivation studies in order to identify the procedures that optimize the advantages of SPECT. We then selectively review the literature in order to illustrate how well neuroactivation studies have succeeded in doing this. To do this effectively it is necessary to consider how well the design of the neuroactivation tasks enables one to identify the neural correlates of specific cognitive processes. Although not exhaustive, this review includes the majority of SPECT neuroactivation studies because only a few have been carried out that focus on the identification of the neural processes that underlie normal cognitive function. While some of the studies have involved patients, the majority of these have involved comparisons of patients with normal controls which have yielded interesting data concerning the neuroactivation of normal cognitive processes. We consider studies that have examined normal subjects alone, patients alone, and both patients and normal subjects before describing the neuroactivation studies that we have performed using the SME 810 Novo scanner (Strichman Medical Equipment, Inc) in Glasgow. We consider that our work with this scanner exemplifies how effective SPECT can be in neuroactivation research.

\section{Optimal and sub-optimal SPECT neuroactivation procedures}

In general, the SPECT technique adopted in neuroactivation studies has varied in several ways: the nature of the SPECT camera used; the radiopharamaceutical used; the procedure (i.e., the method and time over which scans were acquired); and the method used to analyse the data.

Most SPECT cameras are designed to image any part of the body rather than being dedicated to one particular region (e.g., brain). For this reason the position of the camera relative to the source may not always be that which is optimal for maximum sensitivity and spatial resolution. Furthermore, most SPECT cameras are either single or double-headed rotating systems (i.e., have only a relatively low active detector area) which may produce adequate sensitivity and resolution for most clinical investigations, but would not necessarily acquire enough data for accurate neuroactivation studies. By contrast, dedicated brain imaging SPECT systems (such as the SME 810 Novo Tomograph, Strichman Medical Equipment Inc.) can yield sensitivity and resolution comparable to less sensitive PET systems. This is because these kinds of system are designed with multiple detectors and ensure maximal proximity of these detectors to the source. These two factors significantly increase the amount of data acquired at any point in time.

Apart from any general issue such as availability, the radiopharmaceutical used should obviously be that which best serves the aims of the investigation. With respect to neuroactivation studies, it is worth clarifying these aims. It was originally assumed that one important feature of the isotope is that it has a very short half-life, thus allowing for multiple scanning in a single session comparable to most PET designs. As discussed above, however, this feature is not crucial to the design of the study in terms of the questions it can answer since the accuracy of co-registration of scans acquired over time can be reasonably independent of the initial separation between scans. From a practical point of view, however, multiple scans would be beneficial as they allow more data acquisition in a single session. The most extensively used radiopharmaceutical for SPECT investigations is Technetium-99m hexamethylpropyleneamine oxime ( $\left.{ }^{99 \mathrm{~m}} \mathrm{Tc} \mathrm{HMPAO}\right)$ and we will argue that the combination of this with a high resolution SPECT system is the optimal set-up for SPECT neuroactivation studies. ${ }^{99 \mathrm{~m}}$ Tc HMPAO is administered intravenously through a forearm cannula, and reaches the brain after a few seconds whereupon $5 \%-6 \%$ of the total dose becomes trapped in brain tissue. ${ }^{99 \mathrm{~m}} \mathrm{Tc}$ HMPAO is particularly suited to neuroactivation studies since once through the blood brain barrier, its lipophilicity changes and it is trapped such that it does not significantly redis- 
tribute. Therefore, the levels of ${ }^{99 \mathrm{~m}} \mathrm{Tc}$ HMPAO in the brain tissue remain relatively constant for up to eight hours following administration. The trapping mechanism (unique to SPECT) of ${ }^{99 \mathrm{~m}}$ Tc HMPAO allows a cognitive activation task to be carried out away from the scanner, in more natural and appropriate conditions, during which the ${ }^{99 \mathrm{~m}}$ Tc HMPAO dose is administered. The scan can then be carried out later, at a time only limited by the half-life of the nuclide (six hours) and will produce an image of the pattern of blood flow that occurred in the brain at the time of injection. The long half-life of ${ }^{99 \mathrm{~m}} \mathrm{Tc}$ HMPAO is also the source of one of SPECT's perceived limitations; intervals between scans of different cognitive conditions must be at least 24 hours. Each cognitive condition, therefore, requires its own scanning session.

Some studies have tried to overcome this perceived limitation by using short half-life isotopes such as ${ }^{133}$ Xenon $\left({ }^{133} \mathrm{Xe}\right)$. This radiopharmaceutical can be used for multiple scans (i.e., multiple conditions) run approximately one hour apart (e.g., see [4]) or less. However, the relatively low spatial resolution that can be achieved with ${ }^{133} \mathrm{Xe}$ presents a major limitation. The resolution of ${ }^{133} \mathrm{Xe}$ is much poorer than that of other radiopharmaceuticals (e.g., ${ }^{99 \mathrm{~m}} \mathrm{Tc}$ HMPAO) for two reasons. First, its short half-life reduces the possible scanning time and therefore the data acquired (i.e., reducing sensitivity) which may be increased by using wider holed collimators but that would, in turn, reduce the spatial resolution. Second, the energy level emitted by ${ }^{133} \mathrm{Xe}$ is lower than that of ${ }^{99 \mathrm{~m}} \mathrm{Tc}$ HMPAO which introduces a greater scatter component within the signal, further reducing resolution. It is also the case that there is a high absorption of low energy photons that are emitted from the middle of the brain which would particularly reduce resolution of activations in subcortical structures. Since there is a trade-off between brain coverage and resolution, obtaining the best resolution achievable with ${ }^{133} \mathrm{Xe}$ means that only a few brain slices can be imaged. High resolution information about the whole brain is, therefore, impossible to obtain.

Various efforts have been made to attempt to model the PET design with SPECT in order to increase the number of cognitive conditions that can be imaged. Two approaches have focused particularly on the desire to acquire multiple scans in a single session. First, the split-dose procedure which typically involves the administration of two unequal doses of the radiopharmaceutical [28], although equal doses can be used. In this way, a proportion (e.g., 25\%) of the full dose is administered for scan one and data acquisition time is extended to compensate for the low dose. The remainder of the dose can then be administered immediately following the first scan, during which a different cognitive activity is carried out, and the subject is then scanned again. The second approach involves dual isotope imaging [22] whereby two different radiopharmaceuticals are administered in the same way as the two unequal doses in the split-dose procedure. Therefore, two scans are acquired one following the other, each of which could potentially image the differential blood flow patterns produced by two cognitive tasks. While this approach appears promising for SPECT activation work, its original development and evaluation [22] does not appear to have been applied to neuroactivation studies. While both of these procedures offer some chance of acquiring multiple scans they are perhaps limited in their spatial resolution since, particularly with the splitdose procedure, there is considerable background noise residual from the first activation, and this would be sufficient to significantly reduce the sensitivity of the procedure. Neuroactivation paradigms generally produce changes in blood flow of the order of $1 \%-2 \%$ and accurate detection of this level of change probably requires a higher level of sensitivity than can be reliably offered by the split-dose procedure. Although this procedure may be less noisy when a low first scan dose is used and robust activations may be detectable using voxel-wise statistical analysis techniques such as statistical parametric mapping (SPM) [7], these possibilities have not yet been properly explored. It is also important to point out that both these approaches only provide two scans per session whereas most PET designs have involved at least four different cognitive conditions to be imaged in one, albeit long, session.

An alternative approach to this limitation has been developed, evaluated and adopted by Barnes et al. [1] and Montaldi et al. [19]. This procedure does not focus on multiple scans per session but on increasing the number of scans that can be acquired with a ${ }^{99 \mathrm{~m}} \mathrm{Tc}$ HMPAO dose split equally across sessions. We found that reducing the dose administered for each condition but increasing the scan acquisition time allowed us to successfully image four different cognitive conditions (each scan separated by a minimum of 24 hours). It is important to remember that this four-condition procedure can only be properly carried out with a multidetector, section scanner rather than a rotating camera and with radiopharmaceuticals that have long enough half-lives (e.g., ${ }^{99 \mathrm{~m}} \mathrm{Tc}$ HMPAO). Use of both appropriate scanner and radiopharmaceutical is necessary to 
enable data acquisition time to be extended sufficiently to achieve the required level of sensitivity.

The requirements for the analysis of SPECT data are similar to those for PET. For this reason neuroactivation data acquired from SPECT imaging has been successfully analysed using SPM [18] which was originally developed for PET analysis [7]. While SPECT data can be analysed using SPM it can also be analysed using a regions of interest approach (ROI). Indeed, it is probably the case that due to the richer data acquired with SPECT's longer acquisition time, SPECT is more suitable to this form of analysis than is PET. Accuracy with either technique (SPM or ROI), however, requires maximal sensitivity and resolution and both techniques have advantages and disadvantages (for a discussion see [18]).

\section{SPECT activation studies of cognitive function}

\subsection{SPECT neuroactivation studies with normal subjects alone}

One of the few examples of work focused on normal subjects has been provided by Goldenberg and his colleagues (for example [9]). These workers used ${ }^{99 \mathrm{~m}} \mathrm{Tc}$ HMPAO mainly to explore visual imagery processes. In an initial study [8], they compared the activations produced by resting, encoding meaningless "words", encoding concrete words, or encoding abstract words. With the concrete words, subjects were either instructed to encode in the way they chose spontaneously or to use visual imagery. Relative to the three other conditions, encoding concrete words activated the inferior temporal and occipital regions bilaterally.

In subsequent work, Goldenberg and his colleagues [9] used two different comparisons to identify the activating effects of using visual imagery. First, they compared activations produced by judging the correctness of high and low imagery sentences, and second, they compared counting the corners of letters with rehearsing the alphabet. The second comparison was not successful in isolating meaningful activational effects, but it was found that subjects who reported more vivid imagery in the corner counting condition showed more activation in inferior temporal and occipital regions. Importantly, these regions were also activated more when judging the correctness of concrete relative to abstract sentences. The results found by this group in later experiments did not, however, agree in all respects with these earlier findings. Thus, Goldenberg et al. [11] found that although judging the correctness of high imagery sentences relative to low imagery sentences produced more inferior occipital activation, it produced less inferior temporal activation.

Several points about this particular study are worth noting. First, it was done very early, well before functional imaging with PET had become an established procedure with normal subjects. Second, for this reason, it used a ROI approach in which the regions were drawn using a template rather than individual MRI scans. As with most ROI approaches, the regions did not cover the whole brain so the method could not be exploratory and hence had to depend on having reasonably well supported hypotheses about which brain regions are likely to be activated by using visual imagery. Third, and relatedly, most of their studies involved an analysis which depended on inter-subject comparisons rather than the intra-subject comparisons that were shortly to become standard in most neuroimaging studies. The advantage of intra- relative to inter-subject comparisons is, of course, that biological variability across subjects is removed as a source of noise in the analysis.

We can also use Goldenberg and his colleagues's series of studies to illustrate several further points. First, the lack of consistency across their studies indicates that replication in neuroimaging work is of critical importance, particularly when the underlying hypotheses are only weakly supported. In our view, insufficient attention has typically been given to replication in functional neuroimaging research.

Second, the work nicely illustrates the strategy of using several tasks in order to isolate specific cognitive processes. It is highly unlikely that one subtraction can successfully separate a key process from other processes that often work in conjunction with it, but the use of several comparisons between different tasks stands much more chance of doing so. Only when all comparisons either activate the same regions, or exceptions can be convincingly explained, can one be confident that the activating effects of a specific process have definitely been isolated. It is worth noting here that SPM provides a specific conjunction analysis that allows pairs of tasks to be analysed in a combined fashion such that only those brain regions involved in that single process, common to all subtracted pairs, will be activated significantly.

Third, imagining colours and maps produced similar activations to each other when SPECT was used as the imaging modality [10] whereas when steady state electrophysilogical potentials ("DC shifts") were record- 
ed from the scalp negative shifts were maximal over parietal sites when subjects imagined maps, but were maximal over temporal and occipital sites when subjects imagined colours [30]. This probably means that the SPECT procedure used by Goldenberg and his colleagues was a fairly insensitive and blunt instrument. Part of the reason for this may be that the SPECT study involved inter-subject analyses while the DC shift study involved intra-subject analyses. This may partially account for the increased sensitivity of the latter technique while illustrating the limitations of the inter-subject design. Nevertheless, it is likely that blood flow measures are correlates of neural processes that overlap with, but are not identical with those that correlate with DC shifts, so the two imaging modalities may be sensitive to slightly different neural processes. If so, this could be exploited in future imaging research.

\subsection{SPECT neuroactivation studies with patients alone}

An example of a neuroactivation experiment using SPECT to investigate cognitive function in a patient with an interesting form of neurological damage was a study performed by Cardebat et al. [4] using ${ }^{133} \mathrm{Xe}$. The data were analysed using an ROI procedure in which the ROIs were drawn directly onto the SPECT images. Their patient had semantic dementia characterized by a category-specific deficit in semantic knowledge of animals (the patient's knowledge of objects was relatively preserved). When she successfully processed the semantic features of object names, the patient showed activation in the left posterior and middle temporal cortex. The authors interpreted this activation as encompassing Wernicke's area and argued that it reflected the achievement of a match between auditory-lexical input and semantic knowledge for items with the kinds of multimodal representations that man-made objects have. As expected the patient failed to process the semantic features of animal names successfully and, presumably for this reason did not activate the temporal cortex regions that she activated when she managed to retrieve the semantic features of man-made objects. Instead, her left and right inferior frontal regions were activated. The authors viewed these regions as corresponding to Broca's area and its mirror image in the right hemisphere, and interpreted their activation as reflecting a failed phonological strategy to evoke the visual semantic features which they argue are charactistic of animals.
This study has several serious limitations. First, although SPECT with ${ }^{133}$ Xe enables several scans to be completed within a few hours, due to the properties of the ${ }^{133} \mathrm{Xe}$ isotope, the technique has considerably lower spatial resolution than does SPECT with ${ }^{99 \mathrm{~m}} \mathrm{Tc}$ HMPAO. This means that the general accuracy of the study was very limited.

Second, partly for this reason, blood flow was only measured in a single slice so it was impossible to explore activations except in a very small brain volume although the authors did ensure that the analyzed slice contained only intact neural tissue.

Third, the ROI analysis procedure was anatomically inaccurate because it failed to co-register the SPECT images with individual structural MRIs in order to identify the ROIs as precisely as possible. Moreover, the SPECT images on which the ROIs were drawn would themselves have been of particularly low resolution; this would have reduced further the accuracy of the ROI definition.

Fourth, the psychological design of this study meant that there was no chance of discovering whether retrieving semantic information about man-made objects activates different brain regions from those activated by retrieving semantic information about animals. This is because the patient failed to retrieve animate information. It is generally recognized that to image the activating effects of a process it is wise to get a subject to perform that process! The patient was, in fact, also clearly impaired in her semantic knowledge of manmade object names so the ability to image the retrieval of this kind of knowledge must also have been very limited. It would have been much more useful to have imaged normal subjects who have no problem retrieving both animate and inanimate semantic information in order to see how the activations shown by the patient differ. If this had been done, it is possible that the authors might have found more frontal activations in both conditions in their patient because she would have been trying much harder than normal people to retrieve not only animate information, but also inanimate information. This seems likely given that frontal activations are believed to reflect the organizational processes that are involved when cognitive activity is not automatic.

This last limitation relates to a general issue about whether there are some aims which can only be fulfilled by doing neuroactivation research with patients rather than people with intact brains. There are at least three aims which are certainly best addressed by using patients. First, by imaging patients with appropriately designed studies it is possible to determine whether or 
not there has been re-organization of the brain. This can be achieved by identifying whether or not new brain structures have taken over the mediation of cognitive processes at which a patient is still performing normally or surprisingly well despite damage to structures which mediate the key process(es) in normal people. Clearly, this is only possible to do by investigating brain damaged patients in whom re-organization of cognitive processes may have occurred. Also, the aim can only be achieved when patients are performing the imaged cognitive processes well or even completely normally.

Second, imaging of patients also enables one to explore the causal role played by specific brain structures in producing activations in connected brain regions. For example, if the amygdala modulates the activity of extrastriate neocortical visual regions to emotionally significant visual stimuli via a backprojection mechanism, then patients with selective amygdala damage should not show the appropriate kind of extrastriate modulation to emotionally significant visual stimuli in comparison to neutral visual stimuli. This kind of aim can obviously not be achieved by studies of normal people with intact brains.

Third, patients are also critical for exploring whether specific activations are essential for the successful completion of a particular cognitive function. For example, neuroactivation studies have often found that retrieval of memories activates the precuneus. It could be, however, that such activations play no role in the process of retrieval, but rather reflect processes that are incidentally triggered when memories are retrieved although these processes play no role in retrieval. Whether or not a region must be activated in order for a function to be completed successfully can obviously be best achieved by imaging patients with the appropriate selective brain damage. If the damage disrupts the function which activates the critical structure in normal subjects, then the activation is probably critical to the function, and this can be determined without imaging. However, if the damage does not disrupt the function, one cannot be confident that this proves the activation is incidental to the successful completion of the function because re-organization may have occurred following the brain damage. In order to show that this has not occurred, one needs to show that the patient displays activations in the same structures as normal people when performing the function in question apart from also showing no activation in the damaged region bearing in mind that, in many cases, even high quality structural MRI information cannot necessarily completely discount a region's ability to function.
It should be noted, however, that all three of the above aims, that are critically dependent on using patients, can only be achieved if the activations shown by the patients are compared with the activations produced by using the same cognitive comparisons in normal subjects. This is clearly relevant to the study of Cardebat et al. [4], which is hard to interpret because it makes no comparison with the activations that normal people show relative to a resting baseline when retrieving semantic information under the same conditions as the patient. A similar comment applies to a study of Cardebat et al. [5] which used ${ }^{133} \mathrm{Xe}$ SPECT in an activation study of language processing in a patient with deep dysphasia. Although this patient showed more right middle temporal cortex activation in a semantic word monitoring condition relative to a condition involving passive listening to a foreign language, the authors' interpretation that the patient had shown a compensatory re-organization in which the right hemisphere had taken over concrete word processing can be questioned. The interpretation would have been far stronger if, using the same activation paradigm, it had been shown that this same comparison consistently activates left hemisphere structures in normal people. Even if one wants to plot changes in the brain activation produced by a specific process over time in patients with deteriorating conditions such as dementia or in whom there may be a positive response to drug or other treatments, the interpretation of results should be far easier if it is known what activations this process produces in normal people.

\subsection{SPECT neuroactivation studies with patients and normal subjects}

Even when SPECT neuroactivation studies with patients have also included normal subjects as a comparison group, they have not typically attempted to ensure that patients perform as well as their control subjects on the "active" task. This is well illustrated by comparisons between a baseline state and performance on the Wisconsin Card Sorting Test (WCST) in schizophrenics and normal subjects. Schizophrenics are known to be impaired at this task so it is not surprising that, unlike their normal control subjects, schizophrenics usually fail to show greater frontal activation when trying to perform the WCST (for example, see [6,23]).

Activations produced by memory processing have also been examined in studies using patients and control subjects. An example of such a study is provided by Busatto et al. [3]. These workers used ${ }^{99 \mathrm{~m}}$ Tc HMPAO in a split-dose design (described above). They ex- 
amined the activating effects of recalling the response word of a series of learnt word pairs in medicated schizophrenic patients and a group of age, sex, and handedness matched normal control subjects. The activation produced by this retrieval condition was compared with the activation produced by a baseline condition in which subjects were presented 40 word pairs aurally twice in succession with the instruction of paying attention to the association between the words and repeating the response word. The imaging results were analysed using a region of interest (ROI) approach which included regions in the medial temporal lobes, the cerebellum, the thalamus, and a variety of neocortical sites such as parts of frontal, parietal, and temporal cortex. Relative to the baseline condition, retrieval in the normal subjects produced more activation not only in the left medial temporal lobes (MTL), but also in the left inferior frontal and anterior cingulate cortices as well as in the right cerebellum. This may have been the first functional neuroimaging study to demonstrate successfully that retrieving verbal materials activates the left MTL region as would be expected on the basis of lesion studies of this region.

One of the main aims of the study was to determine whether the schizophrenics would show reduced MTL activation relative to their normal control subjects when trying to recall the response words of word pairs to which they had been exposed earlier. The rationale for this was the observation that schizophrenics typically perform poorly on memory tasks, which are known to depend in part on functional activity in the MTL region. Surprisingly, the levels of activation the patients showed not only in the MTL ROI, but also in the frontal lobe ROIs did not differ from those of their control subjects. This is surprising not only because independent evidence suggests that the MTLs are malfunctional in schizophrenics, but also because the patients' memory performance was significantly worse than that of their control subjects.

This study represents a good example of the effective exploitation of the advantages of SPECT in neuroactivation studies except that the ROI procedure did not co-register SPECT images with corresponding structural MRIs so as to help identify the ROIs accurately. However, two findings of this pioneering study warrant comment about its design from a cognitive point of view. The two findings were that, first, the MTL activation was seen in both subject groups and second, this activation was not reduced in the schizophrenics despite their worse recall.

The first finding is puzzling because the baseline condition used seems to have involved the encoding of the word pairs for which recall was later tested in the other condition. In other words, recall was found to produce more MTL activation than encoding. But this is against the main run of results that has been found with PET studies of memory. These studies usually find that encoding produces more MTL activation than retrieval (see [15]). Although instructions to remember the word pairs were not given during the encoding baseline scanning condition, this should not have mattered because what is encoded determines subsequent memory and subjects should have been attending to and encoding the associative links between the words. Busatto et al. clearly intended that the retrieval condition involved all the processes present during the baseline condition and also the retrieval processes that should have been specific to it. However, it has been argued that encoding more novel materials produces more MTL activation than does encoding familiar materials and the words were more novel during the encoding condition (see [15] for a review). The balance of the neuroactivation literature would, therefore, lead one to expect that, if anything, the baseline condition should have produced more MTL activation than the retrieval condition.

The second finding is also puzzling because if retrieving verbal memories activates the MTL, then one would expect that activation should increase in proportion to the rate at which memories are being retrieved. But this rate was reduced in the schizophrenic patients relative to their control subjects. Schacter and his colleagues [26] have found support for this view that MTL activation increases as retrieval success increases in a study which varied the level of retrieval success in normal subjects. The subjects who retrieved more successfully showed significantly more MTL activation. Nevertheless, a study by Petersson et al. [24] suggests that retrieval of overlearnt materials may activate the MTL less under some circumstances than the retrieval of less well learnt materials. Therefore, both rate of successful retrieval and the difficulty of producing such retrieval may sometimes increase MTL activation. It seems likely, however, that, in the Petersson et al. study, the extent of difference in retrieval success between the two retrieval conditions was far lower than in the Schacter et al. study. Therefore, there may be a complex function linking retrieval success and effort to level of MTL activation, the nature of which is still unknown.

The main findings of the Busatto et al. study are, nevertheless, to some extent in conflict with the literature. They may be correct, but clearly should be repli- 
cated before they can be accepted with confidence. It is also worth noting that the comparison with the encoding baseline is very hard to interpret. There is evidence that encoding which leads to better subsequent memory causes more MTL activation than does less successful encoding (see [2,15,31] for a review). As the schizophrenics' encoding led to less successful retrieval than the control subjects' encoding, one would expect that this condition would give rise to less MTL activation in the patients. This would need to be checked by using a baseline that activates the MTL less and to an equivalent extent in the two subject groups or by using a different baseline condition such as retrieval of semantic verbal associates. In addition, confidence in the reality of the two main findings would be increased if (a) the two scans were completed days apart so the levels of ${ }^{99 \mathrm{~m}} \mathrm{Tc}$ HMPAO would have returned close to baseline levels, and (b) the ROI analysis was compared with a more standard technique such as SPM in order to determine whether both techniques yielded similar activations.

\subsection{SPECT memory neuroactivation studies conducted with the Glasgow brain-dedicated scanner}

In the only study that examined the activating effects of retrieval [17] we attempted to achieve the first of these aims and use a more appropriate baseline in a study which compared the activations shown by amnesic patients and normal subjects when they recalled the response word of previously learnt word pairs. The activations produced by this kind of verbal episodic retrieval were compared with the activations produced by retrieving the verbal semantic associates of similar stimulus words. This is a more appropriate baseline comparison condition because recalling overlearnt semantic associates is not disrupted by MTL damage or any of the lesions that cause amnesia. In other words, semantic retrieval of this kind should produce minimal MTL activation. The comparison is not perfect, however, because all retrieval is also associated with encoding of information into memory (people can subsequently remember what they recalled during scanning). There were 12 normal subjects in the study: six of these learnt the word pairs to a high level whereas the other six received much less training. In the episodic recall condition, subjects were shown the first word of each pair that they had studied and asked to try and recall the word that had gone with it during the study session. In the semantic recall baseline condition, subjects were shown similar concrete words one at a time and asked to produce another word that was semantically linked to the presented word. The two conditions were run in a counterbalanced order several days apart.

The aim was to compare the episodic retrieval activations in a group of subjects with high levels of recall success and a group of subjects with a low level of recall success. It was also intended that the recall of the less successful group of control subjects would match that of the amnesic patients, who received levels of training equivalent to that of the high performing group of control subjects. If the patients showed a different pattern of activation from the low performing normal subjects, this would be evidence that their brains had re-organized. Only if their recall was similar to that of their control subjects could such an inference be drawn. In the same way that the motor activations of a paralyzed person will obviously be abnormal, one would expect the memory activations of someone who cannot remember to be very abnormal.

In this and our subsequent SPECT neuroactivation studies, a head dedicated system with high spatial resolution was used and the data obtained were always analysed using SPM. The results revealed an interesting double dissociation which is very similar to the one reported by Schacter et al. [26]. We found that the normal subjects with good verbal recall showed significant left MTL activation that was greater than that found in the normal subjects with poor verbal recall. These latter subjects did not show significant MTL activation, but they did show significant frontal lobe activation bilaterally that was greater than that found in the normal subjects with good verbal recall. The good recall subjects did not show significant frontal lobe activations. These results strongly suggest that MTL retrieval activations reflect the number of episodic memories that are reactivated in a given period of time whereas activations in at least some frontal lobe regions reflects the degree to which effortful and intentional organizational retrieval search and checking processes have been activated. Such processes would have been activated more in the subjects performing poorly whereas the subjects with very good recall could have relied mainly on automatic retrieval processes that minimally involve the frontal lobes.

The results of the amnesic group remain to be fully analysed with SPM, but we have done a preliminary ROI analysis with individual co-registered MRI scans [16]. This indicates that the activation pattern shown by the patients was not completely identical to that shown by the control subjects who showed equiv- 
alently low retrieval success. The patients showed a greater left lateral frontal cortex activation than their retrieval matched control subjects. This suggests that the patients' neural processing of retrieval may have undergone some re-organization. However, it is also possible that, they may have adopted a different strategy for achieving retrieval because of their longterm experience of having poor memory. This strategic adaptation may have exploited the planning capacity of parts of the left frontal cortex. The possibility is consistent with a meta-analysis of imaging studies of retrieval [21], which concluded that the left pre-frontal cortex is particularly activated when executive processes are required to achieve successful retrieval.

In two other studies, we examined the activations produced by encoding information into memory. Our first study [18] extrapolated from a study of Grady et al. [12] in which the activations produced by trying to remember new faces was compared with the activation produced by judging which of two lower face pictures matched an upper picture. Both studies examined the activations produced by normal subjects. Unlike Grady and her colleagues, we examined memory for novel complex scenes, and also unlike their study, we instructed subjects to encode the pictures in the active encoding condition in a meaningful and associative fashion in which they tried to identify the links between the objects in different parts of each picture. The low level perceptual matching baseline condition was supposed to place similar perceptual processing demands on the subjects, but to discourage meaningful associative encoding. Ten normal control subjects performed both these conditions in a counterbalanced order with several days between the performance of each condition. We examined memory of the subjects for the pictures shown in both conditions in two ways. First, subjects were asked to recall as much as the could from a limited, self-selected subset of the pictures, a kind of retrieval that taps the ability to remember associations between objects shown in individual studied pictures. Recall was close to floor for pictures shown in the perceptual matching condition, but good for pictures shown in the encoding condition. Second, the subjects were given a recognition test for the pictures shown. Recognition was very good and did not differ for pictures shown in the encoding and perceptual matching conditions.

One of the main aims of the study was to determine whether encoding associative features of complex pictures into memory produced MTL activation. In order to do this it was necessary to try and match the level of item (picture) memory shown by the subjects be- tween the encoding and matching conditions, and get better associative memory from the encoding condition. The recognition test tapped item memory, which was matched between the conditions, whereas the recall test tapped associative memory, which was better in the encoding condition. There is a widely held view, based on lesion studies (see [15] for a review) that the MTL is critical for both item memory and memory for associations between items. Although it should, therefore, activate when either items or associations between items are encoded into memory, to show that encoding associations into memory specifically activates the MTL it is necessary to match memory for items across compared conditions so that only associative memory differs. We had, therefore, succeeded in doing this. An SPM analysis showed that the encoding condition produced more activation than the perceptual matching condition in the left anterior MTL (Fig. 1(a)) and left anterior cingulate cortex as well as in the right frontal cortex. The same pattern of activations was also found when an ROI method of analysis was used. The ROI analysis did, however, find that there was also a weak right MTL activation so although the results of the two methods of analysis were closely matched, they did not produce identical patterns of results.

The results of this study have several implications. First, they show that the activations produced by encoding pictures into memory found with SPECT are broadly similar to those which have been found with PET [12]. This shows that the appropriate SPECT system may be as sensitive as PET at detecting the relatively small blood flow changes triggered by cognitive processes. The same conclusion can be drawn from the retrieval activation study.

Second, our results provide support for the view that MTL encoding activations are typically anterior when detected by emission tomography as has been argued on the basis of a meta-analysis by Lepage et al. [13]. When we repeated this study using fMRI [20] we found that the MTL activations detected were also left-sided, but they were in the posterior MTL. Such posterior MTL activations have typically been found when the imaging modality has been fMRI rather than emission tomography (see [27]). Our results also provide strong support for the view that this apparent difference between emission tomography and fMRI may not always relate to differences in the encoding paradigms that have been used with these two imaging modalities, but may relate instead to susceptibility artefact reducing the signal to noise ratio of the BOLD response in the anterior MTL. The lesion literature gives no strong reason for expect- 
ing MTL encoding activations to be either anterior or posterior so it may be that the MTL is activated along its entire length, but slightly more strongly in the anterior portion so that more posterior activations are not always detected by emission tomography. This pattern would be reversed with fMRI because susceptibility artefact reduces signal strength at the anterior end of the MTL.

Third, our results also indicate that broadly similar results are found with different methods of analysis such as SPM and ROI. Although cross-validation is not the same as validation, it is essential that different methods of analysis with equivalent face validity should give broadly the same results otherwise the data would be uninterpretable. Where the methods give different results further evidence needs to be sought in order to confirm which result is correct. For example, only the ROI method found that picture associative encoding produced right MTL activation. Although such rightsided activation might well be expected to be produced by the encoding of complex visual materials such as scenes, it could be that closely equivalent levels of right MTL are produced in the perceptual matching condition so that the activations cancel each other out with some forms of analysis (see [18] for a more indepth discussion of this).

Some more light was thrown on this issue in a second picture encoding activation study that we performed with normal subjects using the same SPECT system and the SPM method of analysis. In this study, we made a number of modifications to what we had done before. First, by halving the dose level of ${ }^{99 \mathrm{~m}} \mathrm{Tc}$ HMPAO for each scan, while extending acquisition time [1], we were able to run the first four condition SPECT neuroactivation study with normal subjects. As PET requires repetition of conditions to achieve the same image quality, this effectively enabled us to image the same number of different cognitive conditions as PET. Second, we modified the encoding condition so that subjects now saw a series of sets of three different pictures. For each set, they had six seconds to decide which of the lower two pictures was thematically most similar to the single upper picture. As they also had six seconds to make their perceptual decision in the perceptual matching condition and there were also three similarly arranged pictures, the two conditions were very alike except with respect to how subjects encoded the pictures. Third, four conditions were needed because in two of them subjects either made thematic judgements or perceptual judgements about pictures with which they were already familiar. In the other two conditions, they made these two kinds of judgement about pictures which they had not seen before. Fourth, associative memory for objects in the pictures was this time assessed by a recognition test rather than a recall test. We also debriefed subjects in order to determine whether or not they had noticed at the time of encoding whether the pictures were novel or familiar.

Apart from replicating the finding of the first study that associative encoding activates the left MTL, this second encoding study had two further aims. First, it aimed to determine whether associative encoding also activates the right MTL when the data are analysed with SPM. Second and more important, it aimed to determine whether novelty detection activates the MTL even when a strenuous attempt is made to match encoding between novel and familiar pictures by giving very specific encoding instructions. It has been argued that the detection of novelty leads to MTL activation, but it remains unclear whether any such effect is caused by novelty detection per se, the attentional orienting that such detection typically produces, or the additional encoding that such detection may produce when encoding is spontaneous (see [15] for a review of these issues). We aimed to minimize differences in encoding between novel and familiar pictures. Also, the demanding nature of the encoding in which subjects would have to engage made it seem likely that attentional orienting to novelty would also be minimized. Finally, we wanted to determine whether encoding familiar pictures would activate the MTL as much as encoding novel pictures. This aim relates to the question of whether encoding into memory activates the MTL regardless of whether subjects perceive the information being encoded as novel or familiar.

The results were clearcut, but surprising. First, only thematic encoding gave rise to significant associative recognition for the contents of the pictures and this encoding produced more left MTL activation than the perceptual matching condition regardless of whether the pictures were novel or familiar (Fig. 1(b)). Interestingly, the activation was relatively posterior although we do not believe that a different part of the MTL was activated in this second study than in the first. Rather, it is likely that activation strengths differed between the studies in anterior and posterior regions of the MTL. Second, when encoding was balanced across comparisons, there was no evidence that novelty of the pictures was associated with significant activation anywhere in the brain, let alone in the MTL. No activation occurred despite the fact that subjects claimed to have detected whether pictures were novel or familiar during the the- 


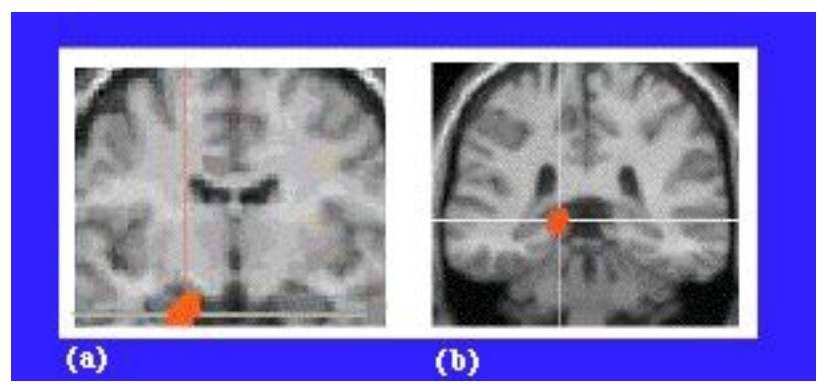

Fig. 1. SPM z-maps of left MTL activations produced by encoding processes carried out during (a) the associative encoding task ( $-16-10-16$, BA 28/35) and (b) the thematic encoding task (-14 -36 0, BA 27/30) when compared to the same baseline perceptual task.

matic encoding and perceptual matching sessions. So the results indicate that novelty detection alone does not produce significant MTL activation. Third, encoding the familiar pictures thematically not only produced as much left MTL activation as did encoding novel pictures, it also produced a right MTL activation. This indicates that when encoding is to some extent controlled and associative memory is effectively produced, the familiarity of pictures does not reduce the role played by the MTL. The greater activation that encoding familiar pictures produced may have occurred because the thematic encoding could be carried out more effectively on slightly familiar relative to completely novel pictures.

Thematic encoding was also found to activate the precuneus bilaterally and the inferior parietal cortex on the left and the extent of these activations correlated strongly with the amount of associative recognition that subjects showed for the pictures. These findings illustrate the importance of using a convergent operations approach which combines lesion with functional neuroimaging research. This is because these activations raise two questions. First, are these activations critical for successful associative recognition of the pictures? As already indicated, one way of answering this question is to find out whether patients with relatively selective damage to the activated structures show impaired associative recognition. Second, what processes do these structures mediate? Again, this question can be addressed by detailed analysis of the cognitive deficits shown by patients who have relatively selective damage to the structures that showed activations during the thematic encoding of the pictures.

The Glasgow series of SPECT neuroactivation studies has used a SPECT system with good sensitivity and spatial resolution. None of the other studies described above used this optimal system. The Glasgow series also used ${ }^{99 \mathrm{~m}}$ Tc HMPAO whereas some of the other studies used radiopharmaceuticals such as ${ }^{133} \mathrm{Xe}$ that fail to exploit maximally the potential advantages of
SPECT. Further, whereas the procedure adopted in the Glasgow series involved up to four intra-subject scan conditions, each separated by at least 24 hours, many of the other studies discussed above used noisier procedures (e.g., inter-subject comparisons or split dose technique) which would have led to reductions in signal sensitivity and spatial resolution. Finally, the Glasgow series used both SPM and MRI-based ROI analyses whereas some of the other studies used less standardized and less structurally accurate analysis procedures.

\section{The future of SPECT neuroactivation research}

The previous section illustrates that SPECT may be as effective as PET when used appropriately in cognitive neuroactivation studies although this capability has rarely been exploited. Equivalent effectiveness will involve the ability to use a SPECT system with high spatial resolution and the ability to image four cognitive conditions. Indeed, in the near future, SPECT systems with spatial resolution of $5 \mathrm{~mm}$ will be available, and $3.5 \mathrm{~mm}$ resolution systems are on the horizon (see www.neurophysics.com). In other words, SPECT resolution in the future will be even more closely comparable to that of PET. It seems very likely, however, that within a few years the modality of choice for nearly all cognitive neuroactivation studies will be fMRI and not emission tomography. Even so, for some time to come, there will be a need to cross-validate fMRI and emission tomography effects. SPECT should play an important role in such cross-validation procedures. Furthermore, there are several specific areas where the use of SPECT in neuroactivation studies is likely to have advantages not only over PET, but also over fMRI.

First, SPECT is very good for imaging patients who are not well oriented and who, therefore, are likely to be become confused in the constraining atmosphere required for fMRI and even PET. This is because sub- 
jects can perform the various challenge tasks in a free environment where the radiopharmaceutical can be administered to them. The unique value in using patients in neuroactivation research has already been discussed. In that earlier discussion, the importance of using normal control subjects as a baseline comparison for the patients was indicated. One of the main aims of using patients is to examine whether re-organization of brain processes has occurred. Future work may develop standardized paradigms, which should mean that it will be unnecessary to use normal subjects repeatedly, but patients' activations will be possible to compare with a standardized normal data base.

Second, for the same reason, SPECT will be the only possible imaging modality in which the activating effects of large scale movements can be examined over a period of about one minute. For example, one could examine the activating effects of planned intentional movements through a complex spatial environment relative to the activating effects of being passively moved through a similar environment. In the foreseeable future, only SPECT will have this capability.

Third, SPECT is the best imaging modality for localizing which brain structures are being disrupted by transcranial magnetic stimulation (TMS). This technique is of great interest because it offers a method for temporarily disrupting electrical activity in the cerebral cortex in a relatively focal way and with minimal discomfort to subjects. TMS can be viewed as a reversible lesioning technique in which it is possible to know within a few milliseconds when the disruption occurred. This means that the technique can be used for determining whether a specific neocortical region plays a critical role in a cognitive function. Not only can it be used for this purpose, but it can even be used for determining when a structure becomes critically involved in mediating a cognitive function. However, there is major problem with TMS. The electrical disruption that it produces is not particularly focal and its localization is hard to determine as it depends on factors such as the orientation of fibre tracts near the cortical surface. Localization may even be influenced by the basal activity of the tissues being stimulated. Ideally, one should use an imaging modality to localize changes in neural activity whilst subjects are engaged in the processes that TMS disrupts. Activity can then be compared whilst doing this task both in the presence of TMS and without it in order to determine exactly what the effect of the stimulation is. Applying the magnetic coils to the same position on the head is far easier to do in an unconstrained environment which is available with SPECT, but not with PET and fMRI.

\section{References}

[1] A. Barnes, D. Dai, D. Montaldi, J. Patterson and D. Wyper, Image Quality versus Statistical Power, Nuclear Medicine Communications 18 (1997), 1155-1160.

[2] J.B. Brewer, Z. Zhao, G.H. Glover and J.D.E. Gabrieli, Making memories: brain activity that predicts whether visual experiences will be remembered or forgotten, Science 281 (1998), 1185-1187.

[3] G.F. Busatto, D.C. Costa, P.J. Ell, L.S. Pilowsky, A.S. David and R.W. Kerwin, Regional cerebral blood flow (rCBF) in schizophrenia during verbal memory activation: a ${ }^{99 \mathrm{~m}} \mathrm{Tc}-$ HMPAO single photon emission tomography (SPET) study, Psychological Medicine 24 (1994), 463-472.

[4] D. Cardebat, J.-F. Demonet, P. Celsis and M. Puel, Living/nonliving dissociation in a case of semantic dementia: a SPECT case study, Neuropsychologia 34 (1996), 1175-1179.

[5] D. Cardebat, J.-F., Demonet, P. Celsis, M. Puel, G. Viallard and J.P. Marc-Vergnes, Right temporal compensatory mechanisms in a deep dysphasia patient: a case report with activation study by SPECT, Neuropsychologia 32 (1994), 97-103.

[6] A.M. Catafau, E. Parellada, F. Lomena, M. Bernardo, J. Pavia, D. Ros, J. Setoain and E. Gonzalez-Monclus, Prefrontal and temporal blood flow in schizophrenia: resting and activation technetium ${ }^{99 \mathrm{~m}}$ Tc-HMPAO SPECT patterns in young neuroleptic-nave patients with acute disease, Journal of $\mathrm{Nu}$ clear Medicine 35 (1994), 935-941.

[7] K.J. Friston, C.D. Frith, P.F. Liddle and R.S.J. Frackowiak, Comparing functional (PET) images: The assessment of significant change, Journal of Cerebral Blood Flow and Metabolism 11 (1991), 690-699.

[8] G. Goldenberg, I. Podreka, M. Steiner and K. Willmes, Patterns of regional cerebral blood flow related to memorizing high an low imagery words-an emission computer tomography study, Neuropsychologia 25 (1987), 473-485.

[9] G. Goldenberg, I. Podreka, M. Steiner, K. Willmes, E.Suess and L. Deecke, Regional cerebral blood flow patterns in visual imagery, Neuropsychologia 27 (1989), 641-664.

[10] G. Goldenberg, I. Podreka, F. Uhl, M. Steiner, K. Willmes and L. Deecke, Cerebral correlates of imaging colours, faces and a map-I. SPECT of regional cerebral blood flow, Neuropsychologia 27 (1989), 1315-1328.

[11] G. Goldenberg, M. Steiner, I. Podreka and L. Deecke, Regional cerebral blood flow patterns related to verification of low- and high-imagery sentences, Neuropsychologia 30 (1992), 581586.

[12] C.L. Grady, A.R. McIntosh, B. Horwitz, J.M. Maisog, L.G. Ungerleider, M.J. Mentis, P. Pietrini, M.B. Schapiro and J. Haxby, Age-related reductions in human recognition memory due to impaired encoding, Science 269 (1995), 218-221.

[13] M. Lepage, R. Habib and E. Tulving, Hippocampal PET activations of memory encoding and retrieval: The HIPER model, Hippocampus 8 (1998), 313-322.

[14] A. Martin, Automatic activation of the medial temporal lobe during encoding: Lateralized influences of meaning and novelty, Hippocampus 9 (1999), 62-70.

[15] A.R. Mayes and D. Montaldi, The Neuroimaging of Long Term Memory Encoding Processes, Memory 7 (1999), 613659.

[16] D. Montaldi, A.R. Mayes, A. Barnes, J. Patterson, D.M. Hadley, J.T.L. Wilson and D.J. Wyper, Use of HMPAO to investigate memory function in patients with amnesia, in: SPECT in clinical neurology and psychiatry.,P.P. De Deyn, ed., John Libbey, London, 1997. 
[17] D. Montaldi, A.R. Mayes, A. Barnes, J. Patterson, D. Hadley and D. Wyper, Contrasting high and low verbal cued recall performance using emission tomography, Neuroimage 9 (1999), S960.

[18] D. Montaldi, A.R. Mayes, A. Barnes, H. Pirie, D.M. Hadley, J. Patterson and D.J. Wyper, Associative encoding activates the medial temporal lobes, Human Brain Mapping 6 (1998), 85-104.

[19] D. Montaldi, A.R. Mayes, A. Barnes, H. Pirie, D.M. Hadley, J. Patterson and D.J. Wyper, Dissociating novelty detection and associative encoding in the processing of complex scenes, Neuroimage 8 (1998), S814.

[20] D. Montaldi, A.R. Mayes, C.E. MacKay, K. Singh. and N. Roberts, Comparison of fMRI and Emission Tomography activations produced by associative encoding of pictures, $\mathrm{Neu}$ roimage 9 (1999), S959.

[21] S.F. Nolde, M.K. Johnson and C.L. Raye, The role of prefrontal cortex during tests of episodic memory, Trends in Cognitive Sciences 2 (1998), 399-406.

[22] D.S. O'Leary, M.T. Madsen, R. Hurtig, P.T. Kirchner, K. Rezai, M. Rogers and N.C. Andreasen, Dual isotope brain SPECT imaging for monitoring cognitive activation: initial studies in humans, Nuclear Medicine Communications $\mathbf{1 4}$ (1993), 397-404.

[23] E. Parellada, A.M. Catafau, M. Bernardo, F. Lomena, E. Gonzalez-Monclus and J. Setoain, Prefrontal dysfunction in young acute neuroleptic-nave schizophrenic patients: a resting and activation SPECT study, Psychiatry Research 55 (1994), 131-139.

[24] K.M. Petersson, C. Elfgren and M. Ingvar, A dynamic role of the medial temporal lobe during retrieval of declartive memory in man, Neuroimage 6 (1997), 1-11.

[25] M.E. Raichle, J.A. Fiez, T.O. Videen, A.M.K. MacLeod, J.V. Pardo, P.T. Fox and S.E. Petersen, Practice-related changes in human brain functional anatomy during nonmotor learning, Cerebral Cortex 4 (1994), 8-26.

[26] D.L. Schacter, N.M. Alpert, C.R. Savage, S.L. Rauch and M.S. Albert, Conscious recollection and the human hippocampal formation: Evidence from positron emission tomography, Proceedings of the National Academy of Sciences USA 93 (1996), 321-325.

[27] D.L. Schacter and A.D. Wagner, Medial Temporal Lobe activations in fMRI and PET studies of encoding and retrieval, Hippocampus 9 (1999), 7-24.

[28] K.J. Schedlack, R. Hunter, D. Wyper, R. McLuskie, G. Fink and G.M. Goodwin, The pattern of cerebral activity underlying verbal fluency shown by split-dose single photon emission tomography (SPET or SPECT) in normal volunteers, Psychological Medicine 21 (1991), 687-696.

[29] J. Talairach and P. Tournoux, Co-Planar stereotaxic atlas of the human brain, Thieme Medical Publishers, Inc., New York, 1988.

[30] F. Uhl, G. Goldenberg, W. Lang, G. Lindinger, M. Steiner and L. Deecke, Cerebral correlates of imaging colours, faces and a map-II. Negative cortical DC potentials, Neuropsychologia 28 (1990), 81-94.

[31] A.D. Wagner, D.L. Schacter, M. Rotte, W. Koutstaal, A. Maril, A.M. Dale and R.L. Buckner, Building memories: Remembering and forgetting of verbal experiences as predicted by brain activity, Science 281 (1998), 1188-1190. 


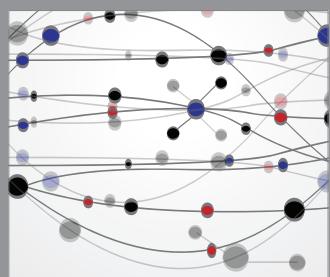

The Scientific World Journal
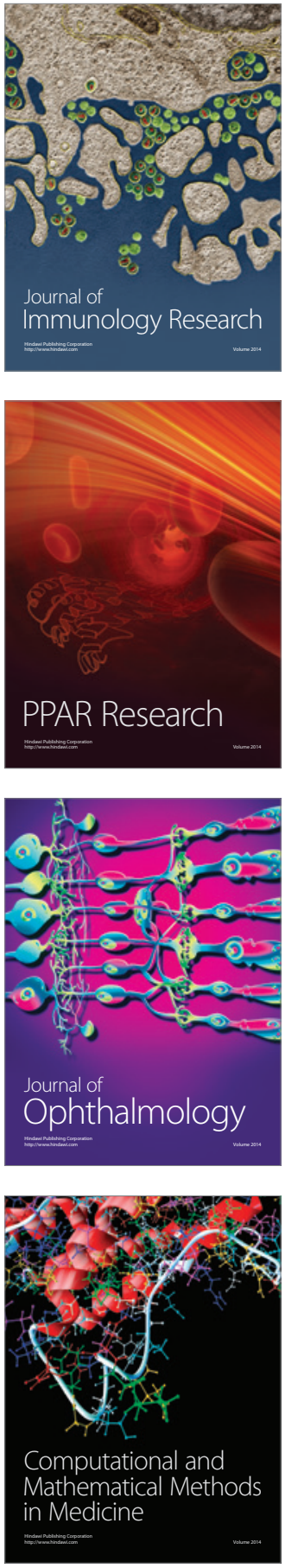

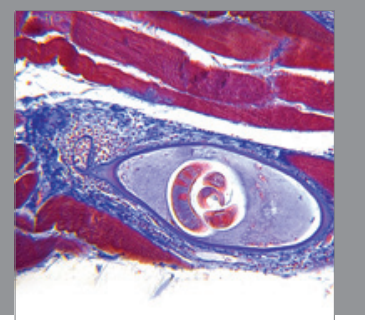

Gastroenterology

Research and Practice
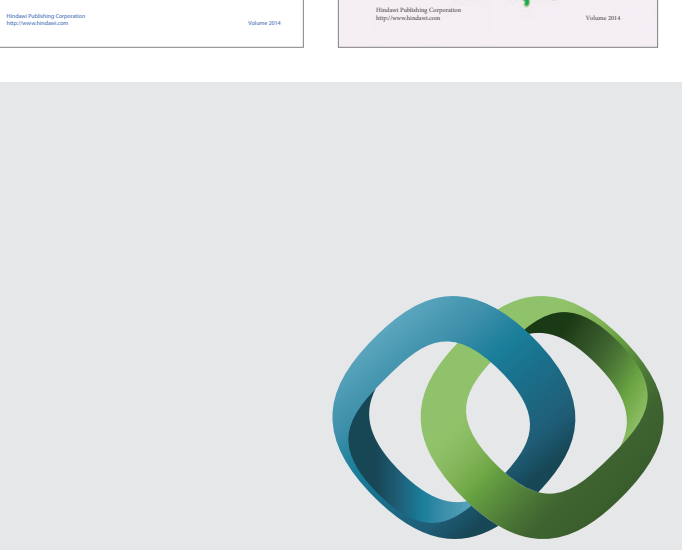

\section{Hindawi}

Submit your manuscripts at

http://www.hindawi.com
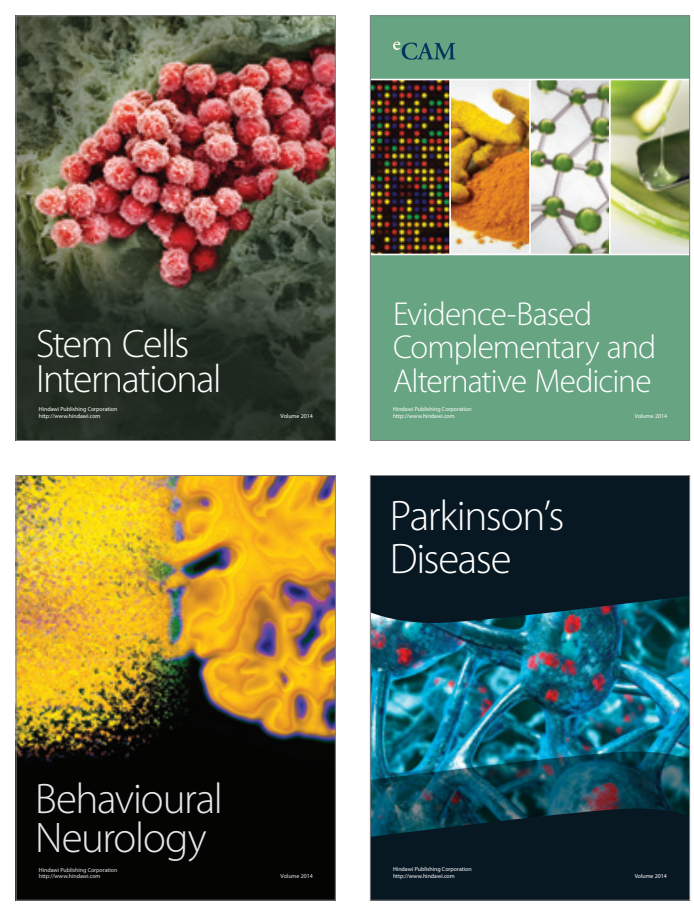

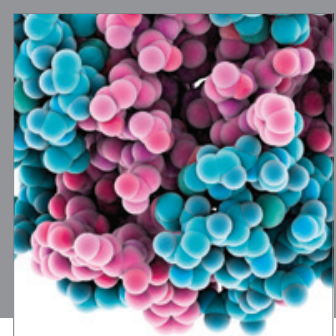

Journal of
Diabetes Research

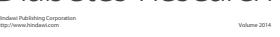

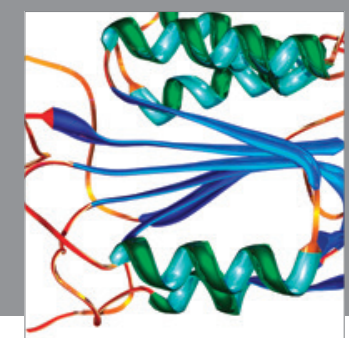

Disease Markers
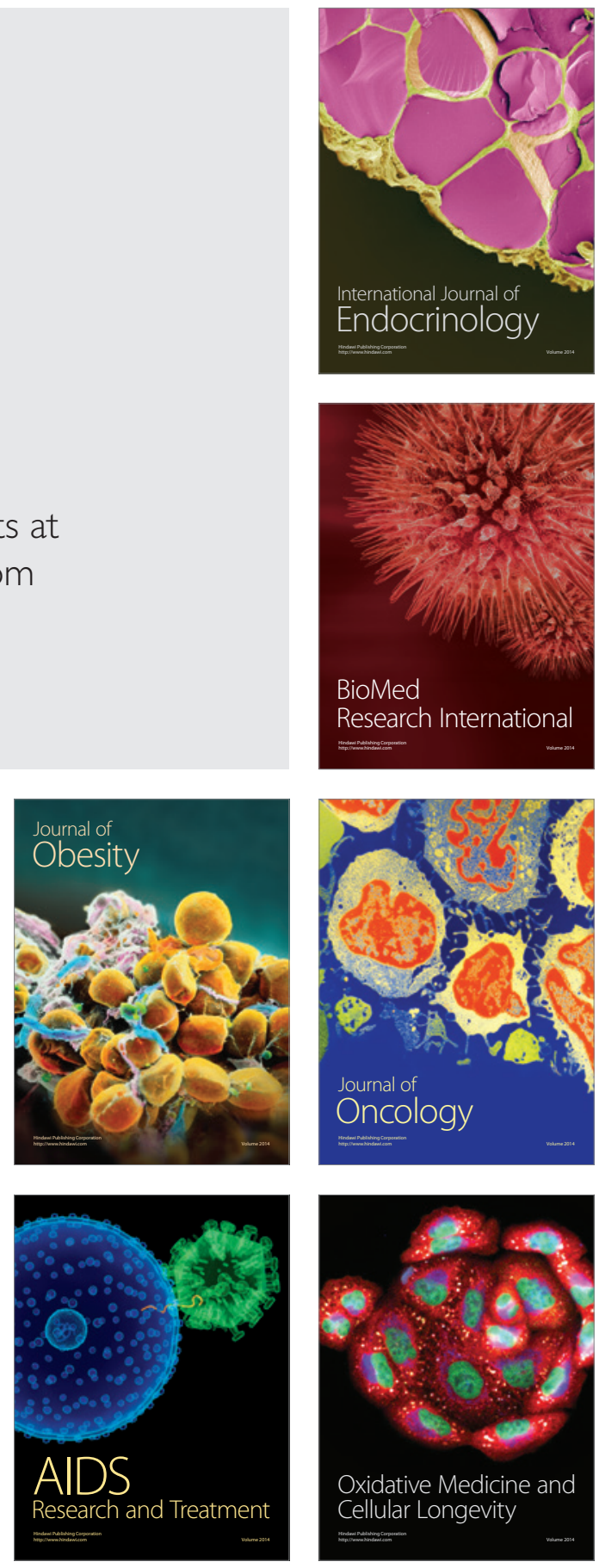